Metal-Catalyzed Asymmetric

Synthesis and

Stereoselective

Reactions

\section{Key Words}

isomerization allylic alcohols rhenium

Highly Selective 1,3-Isomerization of Allylic Alcohols via Rhenium Oxo Catalysis

J. Am. Chem. Soc. 2005, 127, 2842-2843.

\title{
Selective 1,3-Isomerization of Allylic Alcohols via Rhenium Oxo Catalysis
}

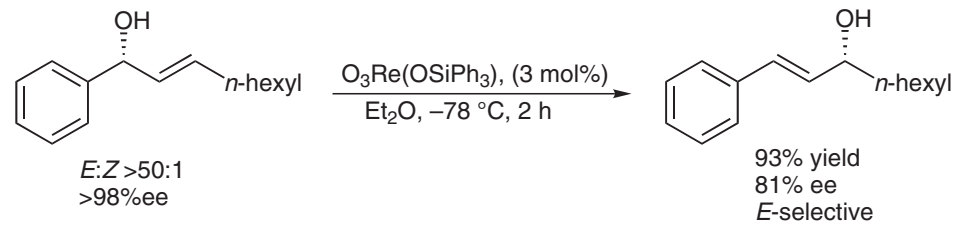

Significance: A protocol featuring mild conditions, short reaction times and low catalyst loading has been developed for the 1,3-isomerization of allylic alcohols using a rhenium oxo catalyst. Enantioenriched starting materials undergo isomerization with only a small loss of optical purity.
Comment: Allylic alcohols are an extremely common feature in natural products as well as intermediates in total synthesis. While the synthesis of these compounds has received much attention, transposition of this functionality under mild conditions with low catalyst loading has not been trivial. While this method enables the selective formation of conjugated allylic alcohols, it gives product mixtures with substrates that lack conjugation. To solve this problem, the authors have developed a method to trap the thermodynamically less stable primary allylic alcohols using N,O-bis(trimethylsilyl)acetamide as an in situ silylating reagent. 